\title{
Doppler assessment of pulmonary haemodynamics in chronic hypoxic lung disease
}

Non-invasive evaluation of pulmonary artery pressure in patients with chronic obstructive pulmonary disease (COPD) has been an important clinical challenge for many years. Several non-invasive methods for measuring pulmonary artery pressure have been developed based on $M$ mode and two-dimensional echocardiography. ${ }^{1-6}$ Whilst all these methods can discriminate mild from severe pulmonary hypertension, they lack sufficient sensitivity to be useful in monitoring the clinical course of patients with COPD in whom pulmonary artery pressures rarely reach very high levels.

However, the development of pulsed and continuous wave Doppler echocardiography has created a new opportunity for assessing right ventricular and pulmonary artery pressures non-invasively with sufficient precision to be useful in routine clinical practice in patients with chronic hypoxic lung diseases.

\section{Technical aspects}

Doppler echocardiography is the most direct and most accurate echocardiographic technique for assessing blood flow. The Doppler signal assesses velocity. Combining velocity with the cross-sectional diameter of the vessel through which the blood is flowing obtained by twodimensional echocardiography provides the basis for quantitating blood flow. In pulsed wave Doppler echocardiography with a single transducer one can measure the velocity in a small range cell at a variable depth along the ultrasound beam, but the maximum velocity that can be measured is limited. Continuous wave Doppler echocardiography with two transducers has no range resolution, but at the same time it has no limit on the maximal velocity measurable. Thus, the two techniques incorporated in any modern echocardiographic machine complement each other, and both methods can be used to measure pulmonary artery pressures non-invasively.

To evaluate pulmonary artery pressures, pulsed wave Doppler echocardiography is used to assess ejection flow velocities in the pulmonary artery. The transducer is placed in the second or third intercostal space along the left sternal border ${ }^{7}$ or subcostally. ${ }^{89}$ The sample volume of the pulsed wave Doppler is positioned just below the pulmonary valve in the middle of the pulmonary artery on the twodimensional echocardiogram. This has been shown to be a crucial step for accurate measurement because velocity measurements may vary with sampling sites, as well as with beam or transducer angulations. ${ }^{111}$

With continuous wave Doppler echocardiography the right ventricular systolic pressure is calculated from the trans-tricuspid gradient. The presence and location of tricuspid regurgitation is first diagnosed when a reverse velocity of flow occurs at the tricuspid orifice and can be followed back into the right atrium, most easily by colour Doppler flow imaging. This new development, based on pulsed mode, which superimposes colour-coded flow pattern on two-dimensional images, has made it possible to map normal and abnormal flow patterns. Continuous wave Doppler recordings of maximal velocity are then obtained from apical, lower left parasternal, and subcostal transducer positions.
The recordings are considered the optimal only after a systematic examination of each transducer position to locate a signal of highest audible frequency, maximal velocity, and clearly definable spectral envelope. The apical transducer position is normally best for this purpose.

\section{Assessment of pulmonary blood flow velocity by pulsed wave Doppler echocardiography}

Quantitative evaluation derived by the pulsed Doppler technique consists of measuring acceleration time (AT, $\mathrm{ms}$ ), defined as the time between the onset of ventricular ejection to peak flow velocity, and right ventricular ejection time (RVET, ms), defined as the interval between onset and termination of flow in the right ventricular outflow tract. In normal subjects there is a discrete velocity envelope in systole with a rounded contour, the maximum velocity occurring in mid systole (fig 1A). In patients with pulmonary hypertension the flow velocity pattern is changed. There is a rapid acceleration of flow velocity, with earlier appearance of its sharp peak and a typical second slow acceleration of flow velocity during deceleration (fig 1B). Factors other than elevated pulmonary artery pressure may be responsible for these changes; reduced capacitance and increased impedance of the pulmonary vascular bed, a prominent systolic reverse flow, ${ }^{12}$ or a pressure wave front which reaches the pulmonary trunk prematurely ${ }^{13}$ are other possible explanations. In addition, decreased stroke volume, ${ }^{7}$ increased venous return, ${ }^{14}$ and even advanced age (Torbicki A, unpublished observation) might also influence the characteristics of the pulmonary flow velocity pattern. Further studies are needed to determine more precisely which mechanism(s) is/are relevant to these changes.

Patients with COPD are more difficult to examine with ultrasound because of hyperinflation, but this method can be used satisfactorily in most of these patients. ${ }^{915}$ With the method described above, it has been shown repeatedly that shortening of AT is the most consistent finding in patients with pulmonary hypertension. ${ }^{7-915-17}$ In addition, the ratio of AT to RVET (AT/RVET) is also useful in diagnosing pulmonary hypertension. By using the lower limit of the normal range of these variables, defined as the mean minus two standard deviations derived from healthy volunteers (AT $=100 \mathrm{~ms}$ ) as a cutoff point, it is possible to diagnose pulmonary hypertension (PAP, mean $>20 \mathrm{~mm} \mathrm{Hg}$ ) with a sensitivity of $71 \%$ and a specificity of $94 \% .{ }^{78}$ In the same studies, AT and AT/RVET were compared with invasively measured mean pulmonary artery pressures. There was a good correlation between these values, with the best correlation coefficient of -0.82 for AT versus PAP mean and -0.85 for AT/RVET versus PAP mean. ${ }^{12}$ Comparison with log PAP mean yielded even better correlations of $r=$ -0.88 and -0.9 , respectively. ${ }^{7} \mathrm{We}^{1618}$ and Kosturakis et $a l^{19}$ have evaluated pulsed Doppler velocity time intervals and ratios in paediatric patients with congenital heart disease and cystic fibrosis and raised pulmonary artery pressures and in control subjects. In all three studies an AT of $<100 \mathrm{~ms}$ identified abnormal pulmonary artery pressures and a significant correlation between the acceleration time and both mean and systolic pulmonary artery pressure was noted. 

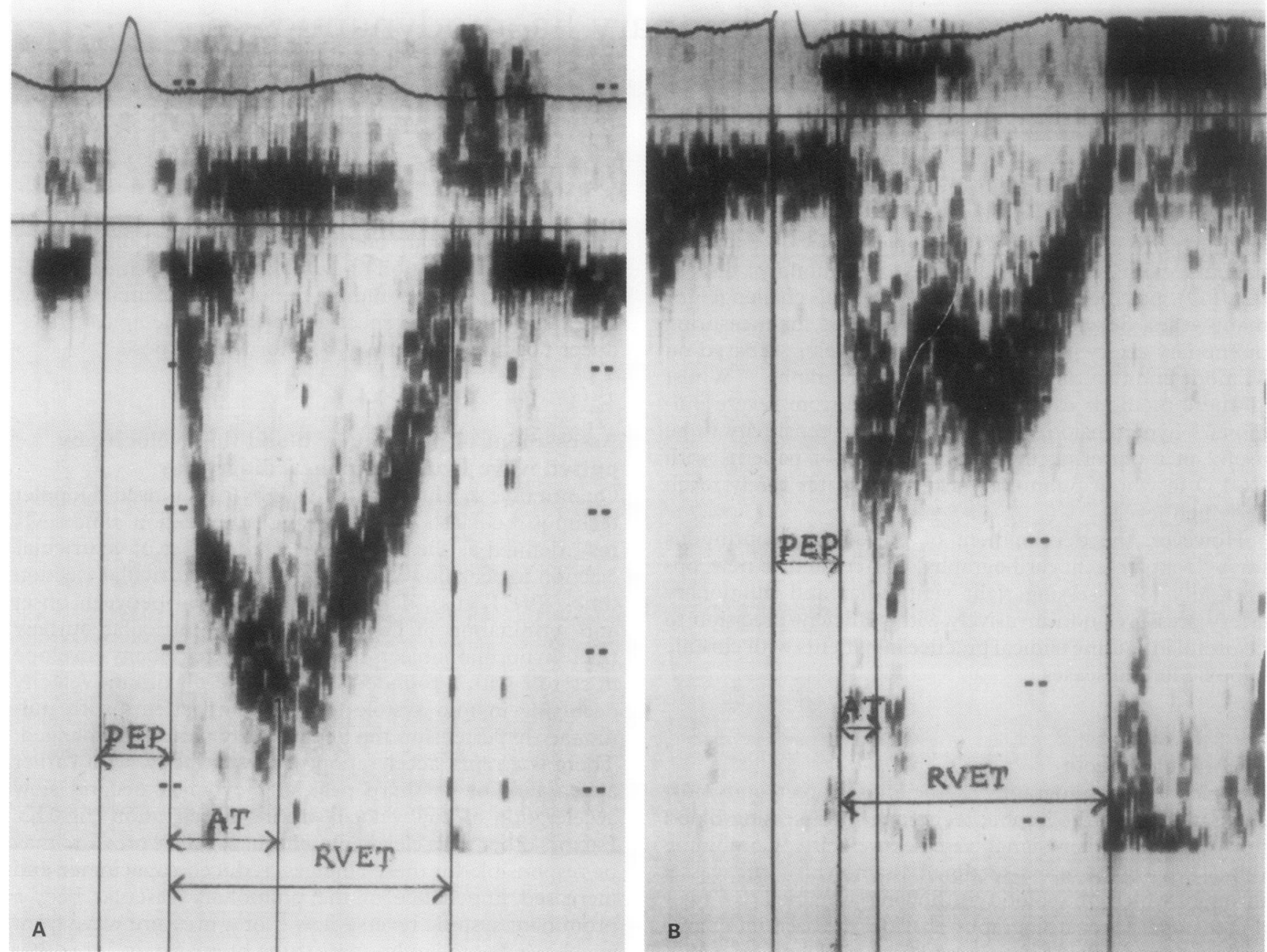

Figure 1 Representative pulmonary flow velocity pattern in (A) a patient without pulmonary artery hypertension and (B) a patient with pulmonary artery hypertension. $P E P=$ pre-ejection period; $A T=$ acceleration time; $R V E T=$ right ventricular ejection time.

All of these studies indicate that the pulsed wave Doppler technique is useful in the diagnosis and evaluation of pulmonary artery hypertension in patients with chronic hypoxic lung diseases. Although this method does not seem as accurate as continuous wave Doppler echocardiography (see below), published data suggest that it may, at least semiquantitatively, estimate pulmonary artery pressure in patients in whom continuous wave Doppler is not feasible because of inadequate imaging or because tricuspid regurgitation is not present. ${ }^{8}$ The technique may also yield prognostic information; Torbicki et al found a shortening of AT below $80 \mathrm{~ms}$ to be an excellent predictor of three year mortality in patients with COPD. ${ }^{20}$

\section{Assessment of trans-tricuspid gradient by} continuous wave Doppler echocardiography

In the presence of tricuspid regurgitation, continuous wave Doppler echocardiography, which is capable of measuring high flow velocities, provides the best method for the noninvasive diagnosis of pulmonary hypertension. Skjaerpe et $a l^{21}$ and others ${ }^{132-24}$ have shown that the gradient across a regurgitant tricuspid valve can be estimated from the peak velocity of the systolic trans-tricuspid jet recorded by Doppler ultrasound. They concluded that prediction of right ventricular systolic pressure should be possible in patients with tricuspid regurgitation by adding the Doppler determined trans-tricuspid gradient to the right atrial pressure estimated clinically.
The best signals are thought to come with the transducer aimed parallel to the direction of the maximal regurgitant blood flow and, with no angle between the ultrasound beam and regurgitant jet, no correction factor is necessary. Thus, the maximal velocity (V) of the regurgitant jet is measured and is inserted into the Bernoulli equation of $\mathrm{TT}-\mathrm{P}=4 \mathrm{~V}^{2}$ (fig 2). Once the trans-tricuspid gradient $(\mathrm{TT}-\mathrm{P})$ is determined, addition of estimated right atrial pressure (PRA) to the Doppler-derived pressure gradient allows an approximation of right ventricular systolic pressure (Prvsyst) by the equation: Prvsyst $=$ PRA + TT $-P$. In the absence of pulmonary stenosis, PRVsyst is equivalent to pulmonary artery systolic pressure. The success of this technique is therefore dependent on (a) the presence of tricuspid regurgitation, obtaining accurate transducer velocity measurements; and (b) the ability to estimate correctly right atrial pressure.

\section{TRICUSPID REGURGITATION}

Tricuspid regurgitation can be recorded with Doppler echocardiography in most patients, with or without pulmonary hypertension. ${ }^{132123-25}$ The feasibility of obtaining this measurement in patients with COPD differs in various studies. Torbitzki et al $^{14}$ showed that tricuspid regurgitation was measurable in only $24 \%$ of patients with COPD, whereas we ${ }^{25}$ and Laaban et $a^{26}$ were able to measure trans-tricuspid gradients in most patients with COPD. Multiple views and contrast enhancement of the con- 


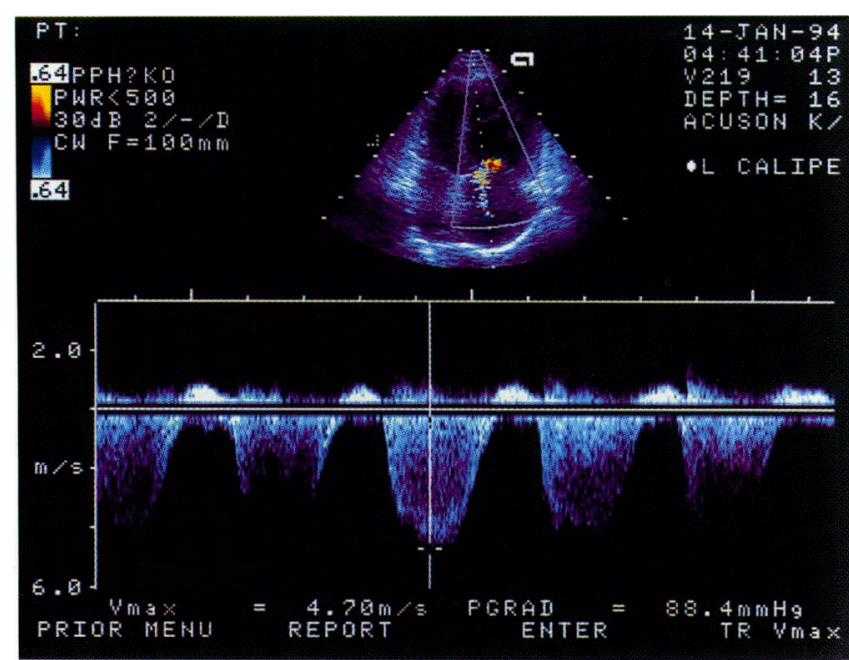

Figure 2 Two-dimensional echocardiography and colour flow imaging of tricuspid regurgitation (above) and continuous wave Doppler ultrasound (below) obtained with the transducer at the apex. The spectral display in the continuous wave mode shows a maximal velocity of $4.7 \mathrm{~m} / \mathrm{s}$. By using the simplified Bernoulli equation a maximal pressure gradient of $88 \mathrm{~mm} \mathrm{Hg}$ between the right ventricle and right atrium is calculated.
Further studies, however, are needed to confirm our results.

Using trans-tricuspid gradient measurements this method provides an accurate non-invasive calculation of pulmonary artery systolic pressure in a variety of patients with and without pulmonary artery hypertension including those with COPD. ${ }^{131521-29}$ Furthermore, the same investigators have shown a good correlation between continuous wave Doppler estimates of right ventricular systolic pressure and right ventricular systolic pressure determined with a catheter in the setting of tricuspid regurgitation with reported correlation coefficients between Doppler and catheter measurements ranging from 0.65 to $0.98 .{ }^{25}{ }^{26}$ Even in patients with mild tricuspid regurgitation, the correlation between non-invasive and invasive measurements of the fall in tricuspid pressure was still good $(r=0.95)$, indicating that different levels of cardiac output do not invalidate the use of this technique. ${ }^{21}$ Furthermore, it has been shown that estimation of the right atrial pressure by various means may not be necessary to obtain reliable results. ${ }^{23}$ Finally, the interobserver variability of the trans-tricuspid gradient measurement has been found to be less than $3 \%$. Thus, in patients with COPD with analysable Doppler tricuspid regurgitant velocities, this method can be used to predict the right ventricular systolic pressure accurately.

tinuous wave Doppler signal by injecting air bubbles intravenously into a peripheral vein have been shown to improve the quality of Doppler signals of tricuspid regurgitation further, which may be very helpful in the presence of hyperinflation. ${ }^{4}$

RIGHT ATRIAL PRESSURE

Several methods exist for the determination of right atrial pressure. In patients with a central venous catheter in situ, right atrial pressure can be measured directly although clearly the need for the catheter makes the method invasive. Simonson et $a l^{77}$ have introduced sonospirometry as a noninvasive way to estimate right atrial pressure based on twodimensional diameter measurements of the inferior vena cava during measured inspiration. However, this method has not been validated nor has it been introduced into clinical practice. An estimation of right atrial pressure can also be made by inspecting the jugular venous pressure. The height of the jugular venous pulse above the sternal angle plus $5 \mathrm{~cm}$, which is the average distance from the right atrium to the sternal angle, provides an estimated right atrial pressure in centimetres of blood. Dividing by 1.3 converts this value to millimetres of mercury. However, there is a poor correlation between this clinical assessment and invasively measured pressures, particularly in patients with COPD who produce large swings in intrathoracic pressures. A fixed estimate of right atrial pressure of $5-12 \mathrm{~mm} \mathrm{Hg}$ has also been proposed. ${ }^{28}$ In fact, a more accurate estimate of right atrial pressure can be obtained by taking into account right atrial dimensions by twodimensional echocardiography and the degree of tricuspid regurgitation by colour Doppler flow imaging, leading to an even better correlation between invasive and noninvasive measurements:

(a) normal diameters of the right atrium and no or mild tricuspid regurgitation - right atrial estimate $=5 \mathrm{~mm} \mathrm{Hg}$;

(b) increased diameter of the right atrium and/or obvious tricuspid regurgitation - right atrial estimate $=$ $10 \mathrm{~mm} \mathrm{Hg}$;

(c) marked increased diameters of the right atrium and/or severe tricuspid regurgitation - right atrial estimate $=$ $15 \mathrm{~mm} \mathrm{Hg}^{25}$

\section{Conclusion}

Various methods of Doppler echocardiography are useful in the analysis of flow dynamics within the heart and the pulmonary circulation in patients with COPD. In addition, to distinguish patients with increased pulmonary artery pressures from those with normal pressures, Doppler techniques provide quantitative methods for estimating pulmonary artery pressures non-invasively. Doppler echocardiography can be performed repeatedly and can thus be used to assess serial changes in the clinical state of a patient or in the response to certain pharmaceutical interventions in the pulmonary vascular bed. ${ }^{30}$ The most useful and accurate method of estimating pulmonary artery pressures in patients with chronic hypoxic lung disease is the systolic trans-tricuspid gradient, calculated from tricuspid regurgitation detected by continuous wave Doppler echocardiography with estimation of the right pulmonary flow analysis using pulsed Doppler techniques.

New contrast materials to enhance the continuous wave Doppler signal and transoesophageal echocardiography may provide even more satisfactory results in the future.

Department of Internal Medicine,

O C BURGHUBER

General Hospital Korneuburg,

Wiener Ring 3-5,

A-2100 Korneuburg,

Austria

1 Bommer W, Weinert L, Neumann A, Neef J. Determination of right atrial 1979;60:91-100.

2 Tsuda T, Sawajama T, Kawai N. Echocardiographic measurements of right ventricular wall thickness in adults by anterior approach. Br Heart f 1980; 44:55-61.

3 Cacho A, Prakash R, Sarma R, Kaushik VS. Usefulness of two-dimensional echocardiography in diagnosing right ventricular hypertrophy. Chest 1983 ; 84:154-7.

4 Himelman RB, Struve SN, Brown JK, Namnun P, Schiller NB. Improved recognition of cor pulmonale in patients with severe chronic obstructive

. Two-dim. using a subcostal Danchin N, Cornette A, Henriquez A, Godenir JP, Ethevenot G, Polu JM, et al. Two-dimensional echocardiographic assessment of the right ventricle in patients with chronic obstructive lung disease. Chest 1987;92:229-33.

7 Kitabatake A, Inoue M, Asao M, Masuyama T, Tanouchi J, Morita T, et al. Noninvasive evaluation of pulmonary hypertension by a pulsed Doppler technique. Circulation 1983;68:302-9. ventricular pressure, followed by the acceleration time from and right ventricular size by two-dimensional echocardiography. Circulation recognition of cor pulmonale in patients with Zenker $G$, Forche $G$, Harnoncourt $K$. Two-dimensional echocardiograph 
8 Burghuber OC, Brunner CH, Schenk P, Weissel M. Pulsed Doppler echocardiography to assess pulmonary artery hypertension in chronic obstructive pulmonary disease. Monaldi Arch Chest Dis 1993;48:121-5.

9 Migueres M, Escamilla R, Coca F, Didier A, Krempf M. Pulsed Doppler echocardiography in the diagnosis of pulmonary hypertension in COPD. Chest 1990;98:280-5.

10 Panidis I, Ross J, Mintz G. Effect of sampling site on assessment of pulmonary artery flow by Doppler echocardiography. Am $\mathcal{F}$ Cardiol 1986; 58:1145-7.

11 Lightly G, Gargiulo A, Krozon I, Politzer F. Comparison of multiple views for the evaluation of pulmonary blood flow by Doppler echocardiography. Circulation 1986;74:1002-6.

12 Okamoto M, Miyatake K, Kinoshita N. Analysis of blood flow in pulmonary hypertension with the pulsed Doppler flowmeter combined with cross sectional echocardiography. Br Heart $\mathcal{f}$ 1984;51:407-15.

13 Yock P, Popp R. Noninvasive estimation of right ventricular systolic pressure by Doppler ultrasound in patients with tricuspid regurgitation. Circulation 1984;70:657-62.

14 Torbicki A, Tramarin R, Fracchia C, Mortara A, Ambrosino N, Pozzoli M et al. Effect of increased right ventricular preload on pulmonary artery flow velocity pattern in patients with normal and increased pulmonary artery pressure. Am 7 Noninvas Cardiol 1994;8:151-5.

15 Torbicki A, Skwarski K, Hawrykiewicz I, Pasierski T, Miskiewicz Z, Zielinski J. Attempts at measuring pulmonary arterial pressure by means of Doppler echocardiography in patients with chronic lung disease. Eur Respir f 1989; 2:856-60.

16 Burghuber O, Salzer-Muhar U, Götz M. Right ventricular contractility is preserved in patients with cystic fibrosis and pulmonary hypertension. Scand 7 Gastroenterol 1988;23:93-9.

17 Marchandise B, De Bruyne B, Delaunois L, Kremer R. Noninvasive prediction of pulmonary hypertension in chronic obstructive pulmonary disease by Doppler echocardiography. Chest 1987;91:361-5.

18 Salzer-Muhar U, Burghuber O, Weissel M, Götz M. Doppler echocardiographic assessment of pulmonary hypertension in adult patients with cystic fibrosis. Prax Klin Pneumol 1988;42:580-2.
19 Kosturakis D, Goldberg S, Allen H. Doppler echocardiographic prediction of pulmonary arterial hypertension in congenital heart disease. $\mathrm{Am} \mathcal{F}$ Cardiol 1984;53:1110-5.

20 Torbicki A, Pruszczyk P, Sliwinski P, Kuch-Wocial A, Hawrykiewicz I, Miskiewicz Z, et al. Pulsed wave Doppler and survival in patients with chronic cor pulmonale. Eur Respir $\mathcal{F} 1993 ; 6: 188 \mathrm{~s}$

21 Skjaerpe T, Hatle L. Diagnosis and assessment of tricuspid regurgitation with Doppler ultrasound. In: Rijsterborgh $\mathrm{H}$, ed. Echocardiology. The Hague: Martinus Nijhoff, 1981:299-304.

22 Hatle L, Angelsen B. Doppler ultrasound in cardiology: physical principles and clinical applications. Philadelphia: Lea and Febiger, 1982:252-64.

23 Berger M, Haimowitz A, Van Tosh A, Berdoff R, Goldberg E. Quantitative assessment of pulmonary hypertension in patients with tricuspid regurgitation using continuous wave Doppler ultrasound. 7 Am Coll Cardiol 1985;6:359-65.

24 Skinner J, Boys R, Hunter S, Hey E. Non-invasive assessment of pulmonary arterial pressure in healthy neonates. Arch Dis Child 1991;66:386-90.

25 Globits S, Burghuber OC, Koller J, Schenk P, Frank H, Grimm M, et al. Effect of lung transplantation on right and left ventricular volumes and function measured by magnetic resonance imaging. Am $₹$ Respir Crit Care Med 1994;149:1000-4.

26 Laaban J, Diebold B, Zelinski R, Lafay M, Raffoul H, Rochemaure J. Noninvasive estimation of systolic pulmonary artery pressure using Doppler echocardiography in patients with chronic obstructive pulmonary pler echocardiography in patien
disease. Chest 1989;96:1258-62.

27 Simonson J, Schiller N. Sonospirometry: a new method for noninvasive estimation of mean right atrial pressure based on two dimensional echographic measurements of the inferior vena cava during measured insraphic measurements of the inferior vena

28 Brecker S, Xaio H, Stoinic B, Mbaissouroum M, Gibson D. Assessment of peak tricuspid regurgitant velocity from the dynamics of retrograde flow. Int $\mathcal{f}$ Cardiol 1992;34:267-71.

29 Abramson S, Burke J, Kelly J, Kitchen J, Dougherty M, Yih D, et al. Pulmonary hypertension predicts mortality and morbidity in patients with dilated cardiomyopathy. Ann Intern Med 1992;116:888-95.

30 Vachiery J, McDonagh T, Moraine JJ, Berre J, Naeije R, Dargie H, et al. Doppler assessment of hypoxic pulmonary vasoconstriction and susceptibility to high-altitude pulmonary oedema. Thorax 1995;50:22-7. 